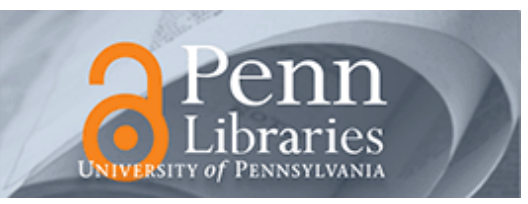

University of Pennsylvania

ScholarlyCommons

\title{
1.05 GHz MEMS Oscillator Based On Lateral-Field-Excited Piezoelectric AIN Resonators
}

\author{
Chengjie Zuo \\ University of Pennsylvania, chengjiezuo@hotmail.com \\ Jan Van der Spiegel \\ University of Pennsylvania, jan@seas.upenn.edu \\ Gianluca Piazza \\ University of Pennsylvania, piazza@seas.upenn.edu
}

Follow this and additional works at: https://repository.upenn.edu/ese_papers

Part of the Electrical and Electronics Commons

\section{Recommended Citation \\ Chengjie Zuo, Jan Van der Spiegel, and Gianluca Piazza, "1.05 GHz MEMS Oscillator Based On Lateral- Field-Excited Piezoelectric AIN Resonators", 2009 Joint Meeting of the European Frequency and Time Forum and the IEEE International Frequency Control Symposium (EFTF-IFCS 2009) , 381-384. April 2009.}

Suggested Citation:

Chengjie Zuo, Jan Van der Spiegel, and Gianluca Piazza. "1.05 GHz MEMS Oscillator Based On Lateral-Field-Excited Piezoelectric AIN Resonators" 2009 Joint Meeting of the European Frequency and Time Forum and the IEEE International Frequency Control Symposium (EFTF-IFCS 2009) (2009): 381-384.

(C)2009 IEEE. Personal use of this material is permitted. However, permission to reprint/republish this material for advertising or promotional purposes or for creating new collective works for resale or redistribution to servers or lists, or to reuse any copyrighted component of this work in other works must be obtained from the IEEE.

This paper is posted at ScholarlyCommons. https://repository.upenn.edu/ese_papers/544

For more information, please contact repository@pobox.upenn.edu. 


\title{
1.05 GHz MEMS Oscillator Based On Lateral-Field-Excited Piezoelectric AIN Resonators
}

\begin{abstract}
This paper reports on the first demonstration of a $1.05 \mathrm{GHz}$ microelectromechanical (MEMS) oscillator based on lateral-field-excited (LFE) piezoelectric Aluminum Nitride (AIN) contour-mode resonators. The oscillator shows a phase noise level of $-81 \mathrm{dBc} / \mathrm{Hz}$ at $1 \mathrm{kHz}$ offset frequency and a phase noise floor of $-146 \mathrm{dBc} / \mathrm{Hz}$, which satisfies the GSM requirements of Ultra High Frequency (UHF) local oscillators (LO). The circuit was fabricated in the AMIS $0.5 \mu \mathrm{m}$ CMOS process, with the oscillator core consuming only 3.5 $\mathrm{mW}$ static power. A simple two-mask process was used to fabricate the LFE AIN resonators from 843 $\mathrm{MHz}$ to $1.64 \mathrm{GHz}$ with high Q (up to 2,200) and kt2 (up to $1.2 \%$ ). This process further relaxes manufacturing tolerances and improves yield. All these advantages make it suitable for post-CMOS integrated on-chip direct $\mathrm{GHz}$ frequency synthesis in reconfigurable multi-band wireless communications.

\section{Disciplines \\ Electrical and Electronics}

\section{Comments}

Suggested Citation:

Chengjie Zuo, Jan Van der Spiegel, and Gianluca Piazza. "1.05 GHz MEMS Oscillator Based On LateralField-Excited Piezoelectric AIN Resonators" 2009 Joint Meeting of the European Frequency and Time Forum and the IEEE International Frequency Control Symposium (EFTF-IFCS 2009) (2009): 381-384.

(C2009 IEEE. Personal use of this material is permitted. However, permission to reprint/republish this material for advertising or promotional purposes or for creating new collective works for resale or redistribution to servers or lists, or to reuse any copyrighted component of this work in other works must be obtained from the IEEE.
\end{abstract}




\title{
$1.05 \mathrm{GHz}$ MEMS Oscillator Based On Lateral-Field-Excited Piezoelectric AIN Resonators
}

\author{
Chengjie Zuo, Jan Van der Spiegel, and Gianluca Piazza \\ Department of Electrical and Systems Engineering \\ University of Pennsylvania \\ Philadelphia, PA 19104, USA \\ \{czuo,jan, piazza\}@seas.upenn.edu
}

\begin{abstract}
This paper reports on the first demonstration of a 1.05 GHz microelectromechanical (MEMS) oscillator based on lateral-field-excited (LFE) piezoelectric Aluminum Nitride (AIN) contour-mode resonators. The oscillator shows a phase noise level of $-81 \mathrm{dBc} / \mathrm{Hz}$ at $1 \mathrm{kHz}$ offset frequency and a phase noise floor of $-146 \mathrm{dBc} / \mathrm{Hz}$, which satisfies the GSM requirements of Ultra High Frequency (UHF) local oscillators (LO). The circuit was fabricated in the AMIS $0.5 \mu \mathrm{m}$ CMOS process, with the oscillator core consuming only $3.5 \mathrm{~mW}$ static power. A simple two-mask process was used to fabricate the LFE AIN resonators from $843 \mathrm{MHz}$ to $1.64 \mathrm{GHz}$ with high $Q$ (up to 2,200) and $\boldsymbol{k}_{t}{ }^{2}$ (up to $1.2 \%$ ). This process further relaxes manufacturing tolerances and improves yield. All these advantages make it suitable for post-CMOS integrated on-chip direct $\mathrm{GHz}$ frequency synthesis in reconfigurable multi-band wireless communications.
\end{abstract}

\section{INTRODUCTION}

Oscillator development based on MicroElectroMechanical Systems (MEMS) has recently drawn significant attention, since MEMS resonators can provide CMOS compatibility and multi-frequency operation on a single chip $[1,2]$. With either electrostatic $[3,4]$ or piezoelectric $[5,6]$ transduction, MEMS resonators have been recently demonstrated to simultaneously attain high quality factor $(Q>1,000)$ and high operating frequencies up to $\mathrm{GHz}$. However, due to the relatively small electromechanical coupling coefficient $\left(k_{t}^{2}<0.6 \%\right)$ and thus large motional resistance, no GHz MEMS oscillator has been demonstrated using laterally vibrating resonators.

In the work reported in this paper, significant progress has been made towards the first demonstration of a $1.05 \mathrm{GHz}$ oscillator based on lateral-field-excited (LFE) piezoelectric Aluminum Nitride (AIN) contour-mode MEMS resonators (Fig. 1). By depositing a piezoelectric AlN layer directly on Silicon wafers and making the film thickness $T$ equal to approximately 0.45 times the desired wavelength of operation [7], $\lambda$, both the material quality (therefore resonator $Q$ ) and $k_{t}^{2}$ have been optimized without being negatively affected by the quality of bottom metal films. In this way, simultaneous high $Q$ (up to 2,200) and $k_{t}^{2}$ (up to $1.2 \%$ ) have been achieved from $843 \mathrm{MHz}$ to $1.64 \mathrm{GHz}$ for LFE AlN resonators without a floating bottom electrode [5]. This solution makes the design of $\mathrm{GHz}$ MEMS oscillators with low power consumption possible. The demonstrated $1.05 \mathrm{GHz}$ oscillator shows a phase noise of $-81 \mathrm{dBc} / \mathrm{Hz}$ at $1 \mathrm{kHz}$ offset frequency with a DC power consumption of $3.5 \mathrm{~mW}$. The integrated circuit (IC) that is wire-bonded to the LFE AIN resonators was fabricated in the AMIS $0.5 \mu \mathrm{m}$ CMOS process.
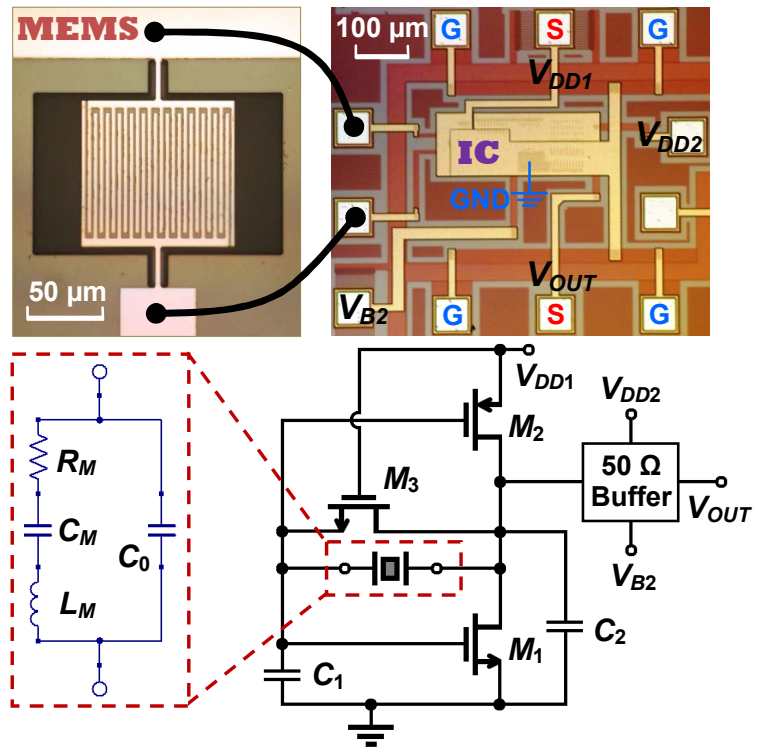

Fig. 1. Micrographs and circuit schematics of the $1.05 \mathrm{GHz}$ LFE AIN MEMS resonator and its wire-bonding to the CMOS IC chip.

\section{PIEZOELECTRIC LFE ALN RESONATOR}

In our previous work, piezoelectric thickness-field-excited (TFE) AlN contour-mode resonators have been demonstrated to have high $Q$ (up to 4,000) in air, low motional resistance $(\sim 25 \Omega)$, and monolithically multiple frequencies of operation $[5,8]$. Based on a similar fabrication process, piezoelectric RF MEMS switches have been monolithically integrated with TFE AlN resonators on the same silicon substrate [9]. TFE AlN resonators have also been used to demonstrate multifrequency oscillators $(176-482 \mathrm{MHz})$ for next-generation reconfigurable frequency reference and timing applications [2]. When the TFE scheme is pushed to higher frequencies $(>\mathrm{GHz})$, the feature size of the electrodes decreases $(\mathrm{GHz}$ 
operation of AlN usually corresponds to few microns for the lithographic patterning of electrodes), which poses a severe challenge for the micro-fabrication. The critical issues include degraded AlN deposition on densely patterned (due to uneven surface) wafers and high vulnerability to alignment errors.

Therefore the idea of introducing LFE resonators without a floating bottom electrode is proposed to solve these problems. By depositing AlN directly on low-roughness Si wafers, the AlN thin film quality can be well controlled and optimized with current-day sputtering techniques. At the same time misalignment errors are greatly relaxed, since only one top metal layer is needed to excite the resonator. Based on this LFE scheme, AlN resonators have been demonstrated up to $10 \mathrm{GHz}$ with the highest $f \cdot Q$ product $\left(\sim 4.6 \times 10^{12} \mathrm{~Hz}\right)$ ever reported for AIN contour-mode devices [6]. For oscillator applications, another important figure of merit (FoM) for resonator design is the $Q \cdot k_{t}^{2}$ product. This has been maximized in this work by making the film thickness $T$ equal to about 0.45 times the desired wavelength of operation [7], $\lambda$, as illustrated in Fig. 2. In this way, simultaneous high $Q$ (up to 2,200 ) and $k_{t}^{2}$ (up to $1.2 \%$ ) have been achieved for LFE AIN resonators from $843 \mathrm{MHz}$ to $1.64 \mathrm{GHz}$, as listed in Table I.

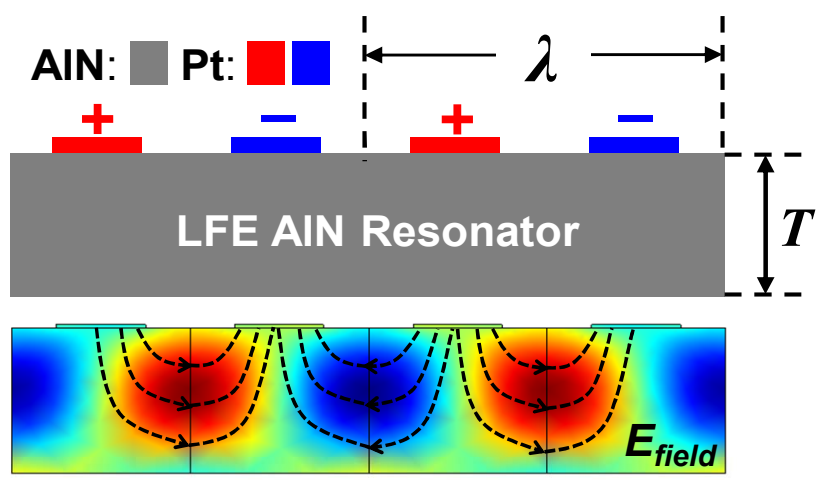

Fig. 2. Cross-sectional schematic and mode shape (displacement profile) of a piezoelectric LFE AlN contour-mode resonator.

TABLE I. EXPERIMENTAL RESULTS OF LFE ALN RESONATORS

\begin{tabular}{|c|c|c|c|c|c|}
\hline $\boldsymbol{f}_{\boldsymbol{s}}[\mathbf{G H z}]$ & $\boldsymbol{Q}_{\boldsymbol{s}}$ & $\boldsymbol{k}_{\boldsymbol{t}}^{\mathbf{2}}$ & $\boldsymbol{R}_{\boldsymbol{M}}[\mathbf{\Omega}]$ & $\lambda[\boldsymbol{\mu \mathrm { m } ]}$ & $\boldsymbol{T}[\boldsymbol{\mu \mathrm { m } ]}$ \\
\hline 0.84 & 1900 & $0.67 \%$ & 425 & 12 & 4 \\
\hline 1.05 & 1450 & $1.20 \%$ & 82 & 8 & 4 \\
\hline 1.17 & 2200 & $0.96 \%$ & 135 & 8 & 3 \\
\hline 1.30 & 1700 & $0.80 \%$ & 143 & 6 & 3 \\
\hline 1.64 & 1450 & $0.39 \%$ & 330 & 4 & 3 \\
\hline
\end{tabular}

$f_{s}$ : series resonant frequency; $Q_{s}$ : quality factor at series resonance; $k_{t}^{2}$ : electromechanical coupling coefficient; $R_{M}$ : motional resistance; $\lambda$ : wavelength of operation; $T$ : thickness of the AlN film

For the fabrication process, only two masks are needed: one for top electrode patterning and the other for AIN etching. Compared with the fabrication of TFE AIN resonators, the LFE scheme greatly reduces the number of steps, eliminates the bottom electrode deposition and the via etching (which has been a significant source of electrical resistance in TFE AIN resonators). Furthermore, due to the intrinsic lower $k_{t}^{2}$ of LFE (less than half of TFE) [7], the motional resistance of each of the sub-resonators (fingers) that form the device is higher than in TFE of comparable dimensions. Therefore, the overall device $Q$ of LFE resonators is less influenced by the electrical loss in the metal electrodes or substrate parasitics. This aspect of LFE AIN resonators relaxes the stringent requirement on metal resistivity for conducting electrodes and reduces their impact on device $Q$.

Similarly, because parasitic electrical loss is negligible for LFE AIN resonators, the traditional Butterworth-Van Dyke (BVD) equivalent circuit model [10], instead of the ModifiedBVD model [2], has been adopted to describe the electrical performance, as shown in Fig. 1. As an example, the measured admittance plot (magnitude and phase), the BVD model fitting curve, and the equivalent circuit parameters of an LFE AlN resonator at $1.17 \mathrm{GHz}$ are given in Fig. 3.
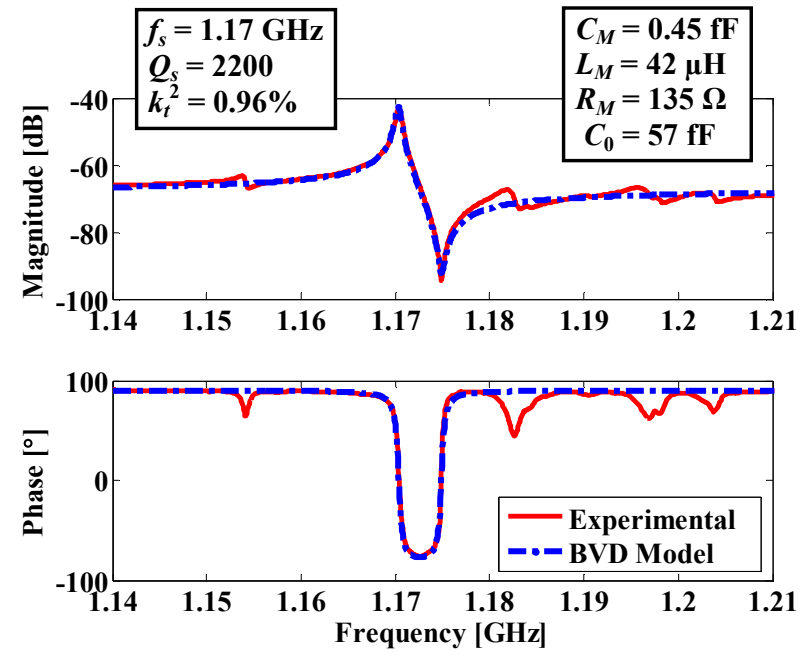

Fig. 3. Measured admittance plot and its BVD model fitting for a piezoelectric LFE AIN resonator at $1.17 \mathrm{GHz}$.

The power handling capability of LFE resonators was also characterized. The electrical response of a $1.17 \mathrm{GHz}$ device was measured for different driving power levels, and the magnitude of admittance is plotted in Fig. 4. As we can see, the critical driving power [11] before bifurcation occurs is between 2 and $4 \mathrm{dBm}$, which roughly corresponds to a critical driving current of $3 \mathrm{~mA}$. Compared with the $222 \mathrm{MHz}$ TFE AIN resonator demonstrated earlier [2], this $1.17 \mathrm{GHz}$ LFE AIN contour-mode resonator has a lower (about 1/3) current handling capability. Intuitively, this can be explained by the smaller volume of the $1.17 \mathrm{GHz}$ resonator. The power handling capability of this device can therefore be improved to the required level by adding more fingers (i.e., resonator volume) to the AlN resonator depending on the specific application of interest. On the other hand, another important factor that can be negatively affecting the power handling capability of the LFE AIN resonators is the non-uniform distribution of the current density, as shown in Fig. 5. This aspect requires further study and it is not addressed in this paper.

All the advantages, related to the elimination of the bottom metal layer deposition, flexibility in material selection (both 
the piezoelectric layer and conducting electrodes), fabrication simplicity, good power handling capability, and high yield, make the LFE scheme extremely suitable for post-CMOS integration applications. The only trade-off consists in the limited frequency range in which the resonators exhibit a high electromechanical coupling $k_{t}^{2}$ for a given thickness $T$ of the piezoelectric material. Nevertheless, assuming a requirement of $k_{t}^{2}>1 \%$, the available wavelength range is between $T / 0.6$ and $T / 0.27$ theoretically [7], which corresponds to a useful frequency band equal to approximately $73 \%$ of the device center frequency for a certain fixed film thickness $T$.

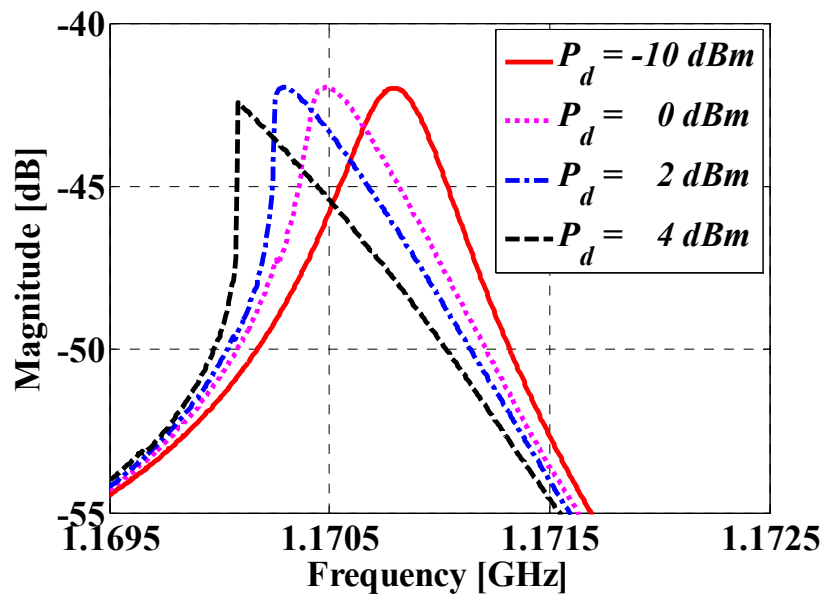

Fig. 4. Measured magnitude of admittance at different driving power levels, $P_{d}$, for the LFE AlN resonator at $1.17 \mathrm{GHz}$.

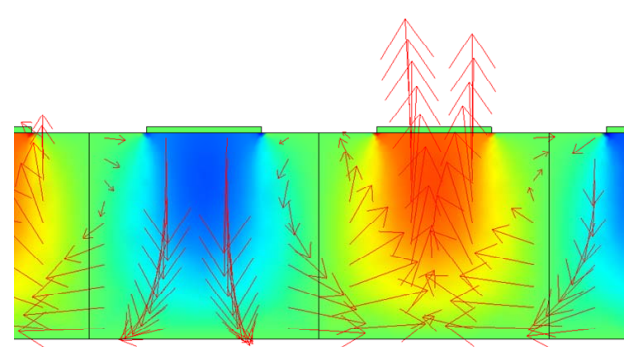

Fig. 5. Distribution of displacement current density (the arrows show both amplitude and direction) in a LFE AIN contour-mode resonator, obtained by COMSOL FEM simulations.

\section{OSCILLATOR CIRCUIT DESIGN}

The circuit topology adopted in this work, shown in Fig. 1, is similar to the Pierce oscillator in [2]. The oscillator core is basically a CMOS inverting amplifier with transistors $M_{1}$ and $M_{2}$ [12]. Transistor $M_{3}$ is biased to be always on and serves as a large resistor to bias the gate and drain voltage of transistors $M_{1}$ and $M_{2}$ at half $V_{D D 1}$. This solution was implemented to minimize resistive loading on the resonator and maximize the allowable oscillating voltage swing. The DC bias current of $M_{1}$ is efficiently reused in $M_{2}$, so that the AC gain of the two transistors adds up. Except for having approximately twice the transconductance $\left(g_{m}\right)$, the small-signal AC analysis of this oscillator core circuit is exactly the same as what has been shown in [2].
The novelty here consists in the idea of implementing a tunable supply voltage $\left(V_{D D 1}\right)$ design. For the oscillator circuit in Fig. 1, the total transconductance can be expressed as:

$$
\begin{aligned}
g_{m}= & g_{m 1}+g_{m 2} \\
\approx & \left|\mu_{n}\right| C_{o x} \frac{W_{1}}{L_{1}}\left(\frac{V_{D D 1}}{2}-\left|V_{T n}\right|\right) \\
& +\left|\mu_{p}\right| C_{o x} \frac{W_{2}}{L_{2}}\left(\frac{V_{D D 1}}{2}-\left|V_{T p}\right|\right)
\end{aligned}
$$

where $\mu_{n}$ is the electron mobility; $\mu_{p}$ is the hole mobility; $C_{o x}$ is the capacitance per unit area of the gate oxide; $V_{T n}$ and $V_{T p}$ are the threshold voltages for NMOS and PMOS transistors, respectively; $W_{1} / L_{1}$ and $W_{2} / L_{2}$ are the effective channel widthto-length ratios for the two transistors. From Equation (1), we infer that the transconductance is linearly proportional to the supply voltage $\left(V_{D D 1}\right)$ for a fixed layout design. Therefore the oscillator core proposed here (Fig. 1) can be effectively used as a tunable amplifier for the reconfigurable multi-frequency oscillator (timing) solution proposed in our previous work $[13,2]$. This solution allows us to optimize the oscillator gain (i.e., power consumption) for each specific frequency of operation of the resonators, instead of being forced to operate with a fixed gain set by the highest frequency of oscillation. By adjusting $V_{D D 1}$, the DC bias current and therefore the $\mathrm{AC}$ gain in the circuit can be set to the point that it is above the critical transconductance for the oscillations to start, so that both the phase noise performance and power consumption can be optimized for each switched-on resonator at a certain operating frequency.

\section{EXPERIMENTAL RESULTS}

The AlN LFE resonators were fabricated in a two-mask micro-fabrication process shown in Fig. 6, while the tunablesupply-voltage oscillator circuit design was implemented in the AMIS $0.5 \mu \mathrm{m} 5 \mathrm{~V}$ CMOS process. The MEMS resonator die was wirebonded to the integrated circuit (IC) chip, and all other electrical contacts were made through the RF and DC probes available in the Desert Cryogenics ${ }^{\circledR}$ TTP6 probe station. The oscillator output was directly probed on chip and monitored through an Agilent ${ }^{\circledR}$ E5052B SSA Signal Source Analyzer. As shown in Fig. 7, the measured phase noise of the $1.05 \mathrm{GHz}$ MEMS oscillator is $-81 \mathrm{dBc} / \mathrm{Hz}$ at $1 \mathrm{kHz}$ offset frequency and as low as $-146 \mathrm{dBc} / \mathrm{Hz}$ when larger than $3 \times 10^{5} \mathrm{~Hz}$, with an output power of $-23 \mathrm{dBm}$. This phase noise performance already satisfies the stringent GSM requirements of Ultra High Frequency (UHF) local oscillators (LO) [14]. Taking into account that the LFE AIN resonators have a power handling capability much larger than $-23 \mathrm{dBm}$, the phase noise floor can be further reduced if the oscillator circuit is designed to operate at a higher power level.

By using this MEMS oscillator technology based on LFE piezoelectric AlN contour-mode resonators, multi-frequency operation over a given frequency range can be realized with much better phase noise performance than conventional LC oscillators. In this way, power-hungry Phase-Locked-Loop (PLL) circuits could be eliminated for frequency synthesis in 
many wireless standards, e.g., GSM, UMTS, CDMA2000, etc. Besides, the extended operating frequency range of these LFE AlN devices up to $10 \mathrm{GHz}$ with high $Q$ and low impedance makes it possible to design novel communication and sensing systems based on non-traditional RF architectures.

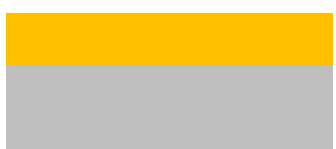

(a)

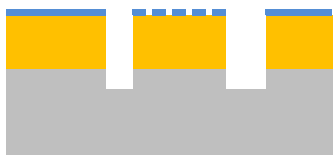

(c)

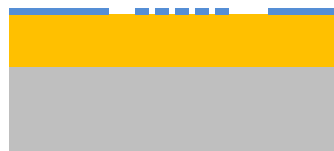

(b)

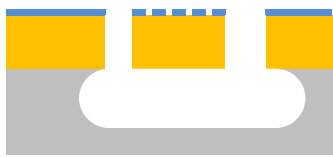

(d)

\section{Si Pt AlN}

Fig. 6. Fabrication process: (a) direct AlN deposition on top of Si wafers; (b) top Pt electrode deposition and patterning; (c) AlN dry etching by Inductively Coupled Plasma; (d) structure release by Si dry etching in $\mathrm{XeF}_{2}$.

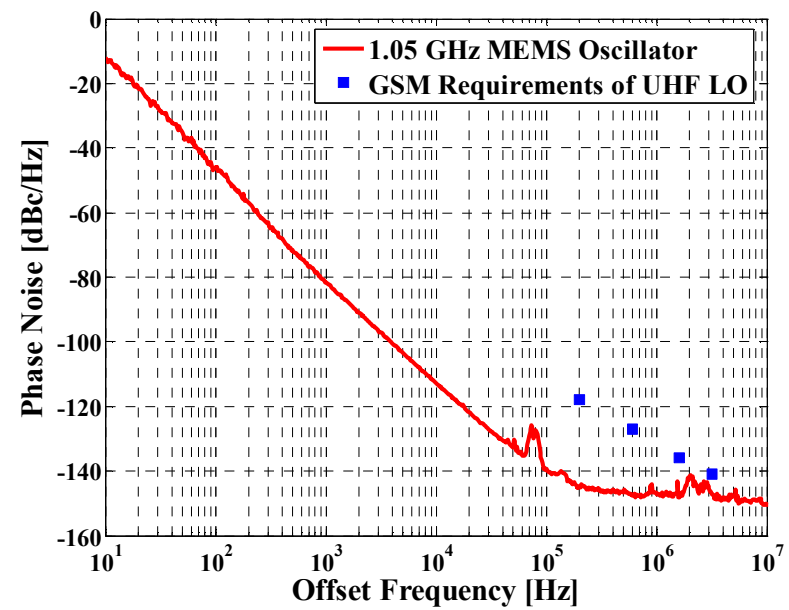

Fig. 7. Measured phase noise of the $1.05 \mathrm{GHz}$ MEMS oscillator based on LFE AlN resonators, with comparison to the GSM requirements of UHF LO.

\section{CONCLUSION}

Design, fabrication and testing of a $1.05 \mathrm{GHz}$ oscillator based on LFE piezoelectric AIN contour-mode resonators have been demonstrated. This is the highest-frequency MEMS oscillator ever demonstrated using laterally vibrating MEMS resonators. The oscillator shows a phase noise performance of $-81 \mathrm{dBc} / \mathrm{Hz}$ at $1 \mathrm{kHz}$ offset frequency and a phase noise floor of $-146 \mathrm{dBc} / \mathrm{Hz}$, which satisfies the GSM requirements for UHF LO. The electromechanical coupling coefficient $\left(k_{t}^{2}\right)$ for LFE resonators has been optimized to attain values up to $1.2 \%$ with simultaneously high $Q$ (up to 2,200) in air. This was made possible by depositing AlN directly on Si wafers and making the film thickness equal to 0.45 times the desired wavelength of operation. In the future, we would like to expand this oscillator technology to microwave frequencies.

\section{ACKNOWLEDGMENT}

The authors would like to thank Nipun Sinha for the help with Pt deposition. We would also like to thank the MOSIS Educational Program for the IC chip fabrication and the staff at the Wolf Nanofabrication Facility at Penn for their support in the MEMS fabrication. Finally, AIN deposition has been performed by Tegal Corporation. The other members of the Penn Micro and Nano Systems Laboratory (PMaNS Lab) also deserve special thanks for precious discussions and help.

\section{REFERENCES}

[1] W.-T. Hsu, "Vibrating RF MEMS for Timing and Frequency References", 2006 IEEE MTT-S International Microwave Symposium, pp. 672-675, Jun 2006.

[2] C. Zuo, N. Sinha, J. Van der Spiegel, and G. Piazza, "Multi-Frequency Pierce Oscillators Based On Piezoelectric AlN Contour-Mode MEMS Resonators", 2008 IEEE International Frequency Control Symposium, pp. 402-407, May 2008.

[3] J. Wang, J. E. Butler, T. Feygelson, and C. T.-C. Nguyen, "1.51-GHz Polydiamond Micromechanical Disk Resonator with ImpedanceMismatched Isolating Support", $17^{\text {th }}$ IEEE International Micro Electro Mechanical Systems Conference (MEMS 2004), pp. 641-644, Jan 2004.

[4] D. Weinstein and S. A. Bhave, "Internal dielectric transduction of a 4.5 $\mathrm{GHz}$ silicon bar resonator," IEEE International Electron Devices Meeting (IEDM 2007), pp.415-418, Dec 2007.

[5] P. J. Stephanou and A. P. Pisano, "GHz Contour Extensional Mode Aluminum Nitride MEMS Resonators", 2006 IEEE Ultrasonics Symposium, pp. 2401-2404, Oct 2006.

[6] M. Rinaldi, C. Zuniga, and G. Piazza, "5-10 GHz AlN Contour-Mode NanoElectroMechanical Resonators", $22^{\text {nd }}$ IEEE International Conference on Micro Electro Mechanical Systems (MEMS 2009), pp. 916-919, Jan 2009.

[7] J. H. Kuypers, C.-M. Lin, G. Vigevani and A. P. Pisano, "Intrinsic Temperature Compensation of Aluminum Nitride Lamb Wave Resonators for Multiple-Frequency References", 2008 IEEE International Frequency Control Symposium, pp. 240-249, May 2008.

[8] G. Piazza, P. J. Stephanou, and A. P. Pisano, "Piezoelectric Aluminum Nitride Vibrating Contour-Mode MEMS Resonators", Journal of MicroElectroMechanical Systems, vol. 15, no.6, Dec 2006.

[9] N. Sinha, R. Mahameed, C. Zuo, M. B. Pisani, C. R. Perez, and G. Piazza, "Dual-Beam Actuation of Piezoelectric AlN RF MEMS Switches Monolithically Integrated with AlN Contour-Mode Resonators", 2008 Solid State Sensor, Actuator and Microsystems Workshop (Hilton Head 2008), pp. 22-25, Jun 2008.

[10] J. D. Larson III, P. D. Bradley, S. Wartenberg, and R. C. Ruby, "Modified Butterworth-Van Dyke Circuit for FBAR Resonators and Automated Measurement System," 2000 IEEE Ultrasonics Symposium, pp. 863-868, Oct 2000.

[11] M. Palaniapan and L. Khine, "Nonlinear Behavior of SOI Free-Free Micromechanical Beam Resonator," Sensors and Actuators A: Physical, vol. 142, no. 1, pp. 203-210, Mar 2008.

[12] Y. H. Chee, A. M. Niknejad, and J. Rabaey, "A Sub-100 $\mu$ W 1.9-GHz CMOS Oscillator Using FBAR Resonator," 2005 IEEE Radio Frequency Integrated Circuits Symposium, pp. 123-126, Jun 2005.

[13] G. Piazza, P. J. Stephanou, and A. P. Pisano, "One and Two Port Piezoelectric Higher Order Contour-Mode MEMS Resonators for Mechanical Signal Processing", Solid-State Electronics, vol. 51, pp. 1596-1608, 2007.

[14] Q. Gu, "RF System Design of Transceivers for Wireless Communications", Springer, 2005. 